Supplement of Hydrol. Earth Syst. Sci., 22, 4281-4293, 2018

https://doi.org/10.5194/hess-22-4281-2018-supplement

(c) Author(s) 2018. This work is distributed under

the Creative Commons Attribution 4.0 License.

(c) (1)
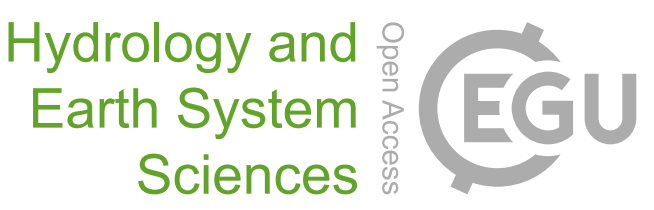

Supplement of

\title{
Technical note: GUARD - an automated fluid sampler preventing sample alteration by contamination, evaporation and gas exchange, suitable for remote areas and harsh conditions
}

Arno Hartmann et al.

Correspondence to: Arno Hartmann (arno.hartmann@kit.edu)

The copyright of individual parts of the supplement might differ from the CC BY 4.0 License. 
$\underline{12 \mathrm{~V}}$

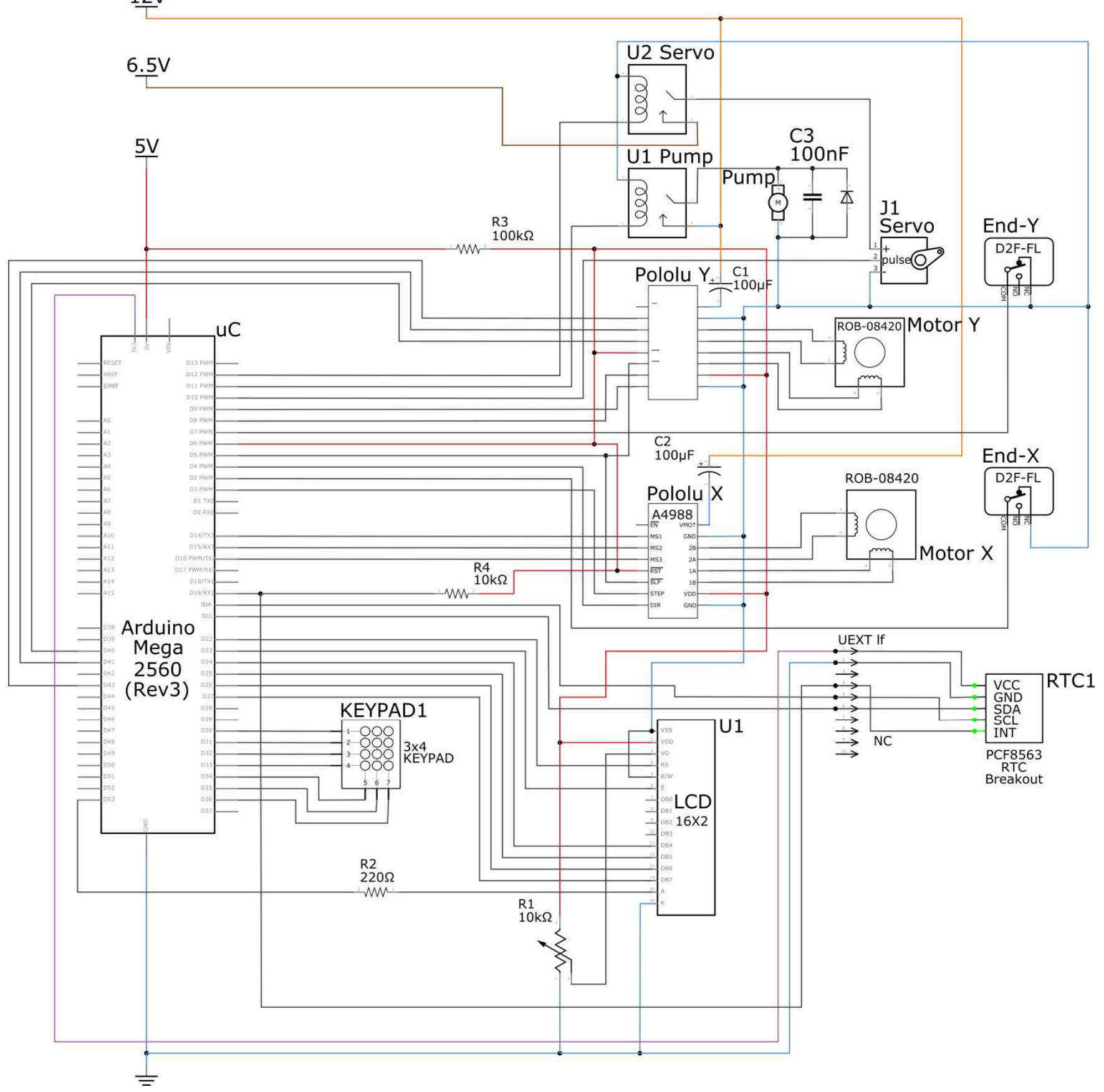

S1: Electrical circuit diagram of the GUARD autosampler. 
The $\delta \mathrm{D}$ results (S2) also confirm the long-term stability of the samples: Again, if the vials were not airtight, evaporation would have led to a preferential removal of isotopically light water molecules from the water samples due to their higher vapour pressure (e.g. Hoefs, 2015) and, consequently, to an increase of the $\delta \mathrm{D}$ value of the remaining water sample over time. Such a positive trend is not present in the $\delta \mathrm{D}$ data and the results from the repeated measurements agree well with the initial ones. The difference in $\delta \mathrm{D}$ values between initial and repeated measurements ranges from - $0.30 \%$ (1t20 and lt23) to $0.70 \%$ (lt02-05), but averages out at $0.0 \%$ over all measurements (median also $0.0 \%$ ) indicating that there is no systemic discrepancy between initial and repeated analyses (S2).

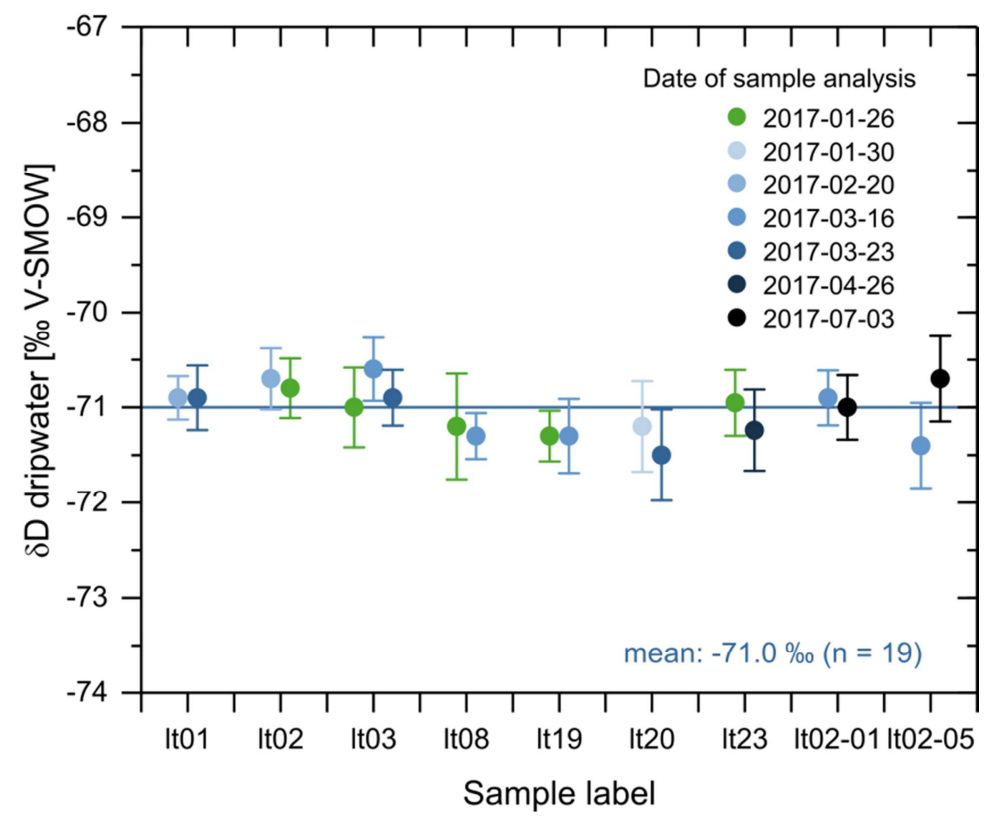

S2: Results of repeated $\delta \mathrm{D}$ measurements (circles in tones of blue) measured in the automatically collected samples together with the original $\delta \mathrm{D}$ data from Fig. 5 (green circles) plotted against their respective label ("It" stands for Laichinger Tiefenhöhle). The darker the tones of blue, the later the respective measurement was repeated. 


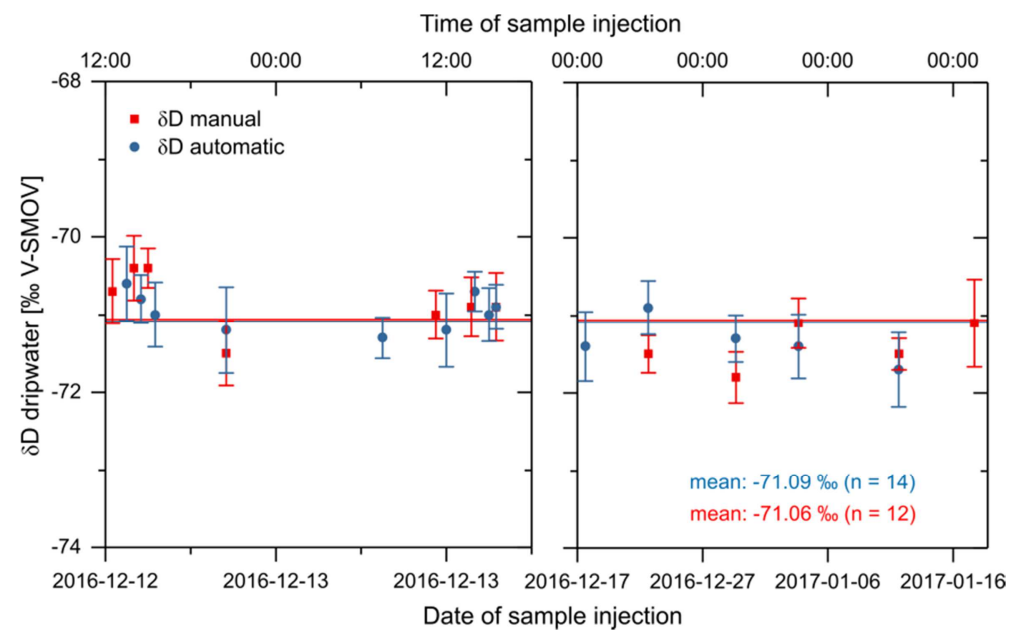

S3: First field testing of the GUARD autosampler: Hydrogen isotope values (indicated as $\delta D$ relative to the international standard V-SMOW) in dripwater samples from a specific drip site in the karst cave „Laichinger Tiefenhöhle“ in the Swabian Alb region, southern Germany. Samples were collected automatically (blue circles) over the course of 33 days (December 13, 2016, to January 14, 2017) and supplemented by 12 samples collected manually (red squares) for comparison of both methods. Error bars represent measurement uncertainty. Blue and red horizontal lines indicate the overall arithmetic mean of each data set. Note the difference in scale of the $x$-axes of the two sub-plots. Not all of the 33 samples were analysed for isotopic composition.

To provide the readership with a notion of the effect of evaporation on the sample $\delta^{18} \mathrm{O}$ values, we have calculated both evaporation and $\delta^{18} \mathrm{O}$ change for the conditions prevalent in our fridge. Despite being set to $8{ }^{\circ} \mathrm{C}$, the temperature in the fridge was measured to be $11.2{ }^{\circ} \mathrm{C}$, relative humidity was $24 \%$ according to measurements. Based on these conditions and assuming an opening of the sample vial of $5 \%$ to imitate a minor lack of airtightness, evaporation was calculated using a formula that has proven adequate for inactive indoor swimming pools that are not influenced by direct sunlight or wind (Smith, Löf and Jones, 1994) using a water density of $1 \mathrm{~g} \mathrm{~cm}^{-3}$ :

$$
\frac{\dot{m}}{A}=\frac{\left(30.6+32.1 * v_{w}\right)\left(P_{w}-P_{a}\right)}{\Delta H_{v}}
$$

where $\dot{m} / \mathrm{A}$ is the evaporation rate $\left[\mathrm{kg}\left(\mathrm{m}^{2} \mathrm{hr}\right)^{-1}\right], \mathrm{v}_{\mathrm{w}}$ is the air velocity over the water surface $\left[\mathrm{m} \mathrm{s}^{-1}\right]$,

$P_{w}$ is the saturation vapour pressure at the water temperature $[\mathrm{mm} \mathrm{Hg}], \mathrm{P}_{\mathrm{a}}$ is the saturation vapour pressure at the air dew point $[\mathrm{mm} \mathrm{Hg}]$ and $\Delta \mathrm{H}_{\mathrm{v}}$ is the latent heat of water at the pool temperature $[\mathrm{kJ} \mathrm{kg}]$.

The $\delta^{18} \mathrm{O}$ value of the residual water remaining at each given time was calculated on the basis of a fractionation factor $\alpha$ between water and vapour according to the following formula (e.g. Clark and Fritz, 1999):

$$
1000 \ln \propto_{\text {water-vapour }}=1.137\left(10^{6} / T_{k}^{2}\right)-0.4156\left(10^{3} / T_{k}\right)-2.0667
$$

where $T_{k}$ represents the temperature of the phase change $[\mathrm{K}]$ and on the following relationship (e.g. Hoefs, 2015):

$$
\frac{R_{w}}{R_{w 0}}=f^{\left(\frac{1}{\alpha}-1\right)}
$$


where $\mathrm{R}_{\mathrm{w}}$ is the isotope ratio of the water at a given time [\%o V-SMOW], $\mathrm{R}_{\mathrm{w} 0}$ is the initial isotope ratio of the water [\%o $\mathrm{V}$ SMOW], and $\mathrm{f}$ is the fraction of the residual water [-]. The results of these calculations (S4) demonstrate that even a small slit in a sample vial's rubber septum equalling only $5 \%$ of the vial's inner cross section leads to a substantial shift towards higher $\delta^{18} \mathrm{O}$ values in the residual water over time. After three months (90 days), for instance, $\delta^{18} \mathrm{O}$ values have risen from $10.1 \%$ by about $1.3 \%$ to $-8.8 \%$. The difference between the lowest and the highest $\delta^{18} \mathrm{O}$ value in Fig. 6 of the manuscript is still below $0.3 \%$, while those data points span a longer period of six months. Most importantly, there is no positive trend in the $\delta^{18} \mathrm{O}$ values in Fig. 6 of the manuscript which illustrates the sample vials are sealed properly, even after sample injection.

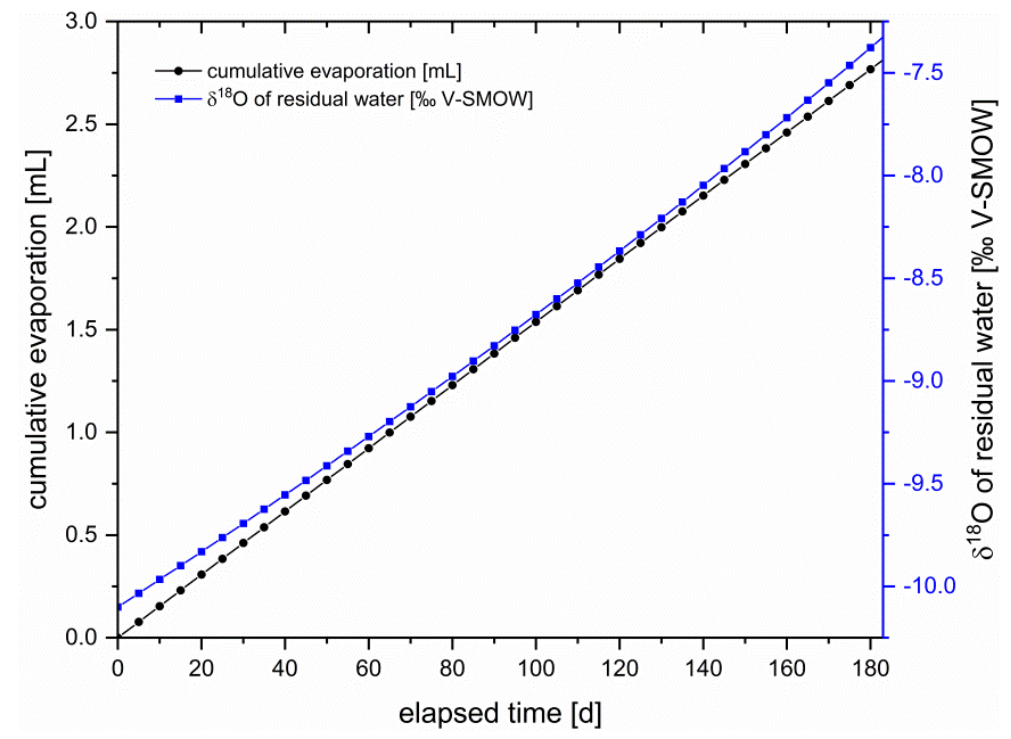

S4: Effect of evaporation on the $\delta^{18} \mathrm{O}$ value of the residual water in a $12 \mathrm{~mL}$ sample vial at a temperature of $11.2{ }^{\circ} \mathrm{C}$ and a relative humidity of $24 \%$. 


\begin{tabular}{|c|c|c|}
\hline COMPONENTS & Description & Dimensions \\
\hline Mechanical & & $\begin{array}{l}\text { Length } \mathrm{x} \text { Width } \mathrm{x} \\
\text { Height }\end{array}$ \\
\hline Z-movement: servo & $\begin{array}{l}\text { Reely }{ }^{\circledR} \text { Standard RS-610 MG, operating voltage } 6.6 \mathrm{~V} \text {, attached to the } \\
\text { Z-slide containing the double-cannula via an elongated hole in the } \\
\text { servo's horn }\end{array}$ & $40 \times 20 \times 42 \mathrm{~mm}$ \\
\hline $\begin{array}{l}\mathrm{X}-/ \mathrm{Y}-\text { movement: } \\
\text { motors }\end{array}$ & $\begin{array}{l}\text { Sanyo Denki }{ }^{\circledR} \text {, bipolar hybrid stepping motors, } 1 \mathrm{~A}, 24 \mathrm{~V}, 1.8^{\circ} \mathrm{step}^{-1} \text {, } \\
0.265 \mathrm{Nm}, 4 \text { wires }\end{array}$ & $42 \times 42 \times 24 \mathrm{~mm}$ \\
\hline Pump & Peristaltic (flexible-tube) pump, model AP-40; operating voltage $12 \mathrm{~V}$ & $55 \times 50 \times 42 \mathrm{~mm}$ \\
\hline
\end{tabular}


S6: Bill of Materials

\begin{tabular}{|c|c|c|c|c|c|c|}
\hline Components & Description & quantity & cost/unit & cost & company & order no. \\
\hline \multicolumn{7}{|l|}{ Mechanical } \\
\hline Casing & $\begin{array}{l}\text { Peli }{ }^{\oplus} \text {, model 1610, heavy-duty, water-tight and airtight, including a valve } \\
\text { for automatic pressure purge }\end{array}$ & 1 & $252.35 €$ & $252.35 €$ & $\begin{array}{l}\text { Waterproof- } \\
\text { Cases }\end{array}$ & - \\
\hline Z-movement: servo & $\begin{array}{l}\text { Reely }{ }^{\circledast} \text { Standard RS- } 610 \mathrm{MG} \text {, operating voltage } 6.6 \mathrm{~V} \text {, attached to the Z- } \\
\text { slide containing the double-cannula via an elongated hole in the servo's } \\
\text { horn }\end{array}$ & 1 & $12.60 €$ & $12.60 €$ & $\begin{array}{l}\text { Conrad } \\
\text { Electronic }\end{array}$ & $1365925-05$ \\
\hline $\begin{array}{l}\mathrm{X} \text {-/Y- movement: } \\
\text { motors }\end{array}$ & $\begin{array}{l}\text { Sanyo Denki }{ }^{\circledast} \text {, bipolar hybrid stepping motors, } 1 \mathrm{~A}, 24 \mathrm{~V}, 1.8^{\circ} / \text { step, } \\
0.265 \mathrm{Nm}, 4 \text { wires }\end{array}$ & 2 & $38.95 €$ & $77.90 €$ & RS Components & $829-3499$ \\
\hline Pump & Peristaltic (flexible-tube) pump, model AP-40; operating voltage $12 \mathrm{~V}$, & 1 & $19.90 €$ & $19.90 €$ & Gemke Technik & APE40CD12V \\
\hline Sample vials & $\begin{array}{l}\text { Labco Exetainer }{ }^{\oplus} 738 \mathrm{~W} \text {, soda glass, } 12 \mathrm{~mL} \text {, flat bottom, height (vial + cap) } \\
\leq 101 \mathrm{~mm} \text {; external } \varnothing \leq 15.5 \mathrm{~mm} \text {; internal } \emptyset \geq 13.2 \mathrm{~mm} \text {; including rubber } \\
\text { septa with a thickness } \geq 3 \mathrm{~mm} \text {; } 48 \text { vials of } 300 \text { in a packaging unit }\end{array}$ & 1 & 22.28 & $22.28 €$ & IVA & IVA738W \\
\hline Tubing & $\begin{array}{l}\text { Deutsch \& Neumann }{ }^{\oplus}, \text { FKM (synthetic rubber, "Viton"), Shore hardness } \\
75 \text {, external } \varnothing \leq 6.2 \mathrm{~mm} \text {, internal } \varnothing 4 \mathrm{~mm}\end{array}$ & 3 & $12.90 €$ & $38.70 €$ & häberle Shop & 9.205765 \\
\hline Double cannula & $\begin{array}{l}\text { Braun Sterican }{ }^{\circledast}, \text { metal, external } \varnothing 0.60 \mathrm{~mm} \text {; length excluding Luer-Lock } \\
\text { connector } 30 \mathrm{~mm}\end{array}$ & 2 & $3.40 €$ & $6.80 €$ & häberle Shop & 7.079505 \\
\hline $\begin{array}{l}\text { Framework for } \\
\text { slide movement }\end{array}$ & Makeblock XY Printer & 1 & $269.95 €$ & $269.95 €$ & Eckstein & MB90014 \\
\hline \multicolumn{7}{|l|}{ Electronic } \\
\hline Battery & $\begin{array}{l}\text { Panasonic }{ }^{\circledR} \text {, valve regulated Pb-acid battery } 12 \mathrm{~V}, 20 \mathrm{Ah} \text {, maintenance- } \\
\text { free, non-spillable, low self-discharge, } 5.8 \mathrm{~kg}, 76 \times 167 \times 181 \mathrm{~mm} \text {; the } \\
\text { sampler can also run on } 12 \mathrm{~V} \text { Li-ion batteries if weight is an important } \\
\text { constraint }\end{array}$ & 1 & $75.03 €$ & $75.03 €$ & Voelkner & S167901 \\
\hline $\begin{array}{l}\text { Microcontroller } \\
\text { board }\end{array}$ & $\begin{array}{l}\text { Arduino }{ }^{\circledast} \text { Mega } 2560 \text { including an Atmel ATmega } 2560 \text { microcontroller } \\
\text { with } 54 \text { digital I/O pins, } 16 \text { analogue inputs, } 6 \text { interrupt inputs, } 4 \text { serial } \\
\text { interfaces, } 1 \mathrm{I}^{2} \mathrm{C} \text { interface and } 4 \text { KB EEPROM memory (non-volatile); } \\
\text { hibernation mode-enabled }\end{array}$ & 1 & $21.99 €$ & $21.99 €$ & Conrad & $1409778-05$ \\
\hline Real-time clock & $\begin{array}{l}\text { RTC PCF } 8563 \text { powered by a separate } 3 \mathrm{~V} \text { lithium button cell battery as a } \\
\text { buffer battery }\end{array}$ & 1 & $10.91 €$ & $10.91 €$ & Conrad & $1195070-05$ \\
\hline Display & Liquid crystal display (LCD) with 2 lines à 16 characters & 1 & $9.87 €$ & $9.87 €$ & Conrad & $183045-05$ \\
\hline \multirow[t]{23}{*}{ Other electronic } & relay module & 1 & $8.52 €$ & $8.52 €$ & Exptech & EXP-R25-187 \\
\hline & drivers for stepping motors & 2 & $7.95 €$ & $15.90 €$ & Exptech & EXP-R25-001 \\
\hline & casing for control panel & 1 & $5.28 €$ & $5.28 €$ & Conrad & $522641-99$ \\
\hline & DC/ DC converter $12 \mathrm{~V}$ & 1 & $12.00 €$ & $12.00 €$ & Conrad & $154170-05$ \\
\hline & DC/ DC converter $5 \mathrm{~V}$ & 1 & $2.65 €$ & $2.65 €$ & Conrad & $157954-05$ \\
\hline & DC/ DC converter 6,5V & 1 & $5.82 €$ & $5.82 €$ & Conrad & $156674-05$ \\
\hline & CR2032 3V lithium button cell battery as a buffer battery & 1 & $2.26 €$ & $2.26 €$ & Conrad & $1086225-05$ \\
\hline & USB service interface FrontCom ${ }^{\circledR}$ Micro IE-FCM-USB-A Weidmüller & 1 & $20.35 €$ & $20.35 €$ & Conrad & $746885-05$ \\
\hline & \begin{tabular}{|l|} 
Membrane keypad Matrix 1 x 12 SU709948 \\
\end{tabular} & 1 & $11.11 €$ & $11.11 €$ & Conrad & $1341283-62$ \\
\hline & 3D print-outs (sample rack, connectors, double-canula adapter) & 1 & $15.00 €$ & $15.00 €$ & - & - \\
\hline & Aluminium slot profiles $20 \times 20 \mathrm{~mm}$ Slot $5(\mathrm{~m})$ & 1 & $2.94 €$ & $2.94 €$ & Motedis & 19586 \\
\hline & Sliding nuts Slot 5100 pieces & 1 & $21.42 €$ & $21.42 €$ & Motedis & 96214 \\
\hline & Screw DIN 7984 M4x10 Slot 5 & 100 & $0.12 €$ & $12.00 €$ & Motedis & - \\
\hline & Bracket 20x40 I-type Slot 510 pieces & 3 & $7.50 €$ & $22.50 €$ & Motedis & 093W202N05 \\
\hline & Swivel Feet. Series 10 PA; foot 40 , threaded rod $5 \times 604$ pieces & 4 & $1.00 €$ & $4.00 €$ & Motedis & - \\
\hline & \begin{tabular}{|l|} 
Miniature sliding rail IGUS drylin TK-04 \\
\end{tabular} & 1 & $10.16 €$ & $10.16 €$ & IGUS & TS-04-07 \\
\hline & CNC Aluminium Servo Horn 60mm for Futaba servos 25 teeth & 1 & $6.90 €$ & $6.90 €$ & Ebay & 251439671553 \\
\hline & Cable gland PG7 Polyamide black (RAL 9005) KSS EGRWW7 water-tight & 1 & $0.34 €$ & $0.34 €$ & Conrad & $533738-05$ \\
\hline & zip ties different sizes 200 pieces & 1 & $3.80 €$ & $3.80 €$ & Conrad & $541665-62$ \\
\hline & USB cable PC/Sampler & 1 & $4.29 €$ & $4.29 €$ & Conrad & $1592198-62$ \\
\hline & Merck $^{\circledR}$ silicone grease for sealing 100gr. & 1 & $68.70 €$ & $68.70 €$ & häberle Shop & 1.07746 .0100 \\
\hline & Hose fitting, straight, 4040 & 10 & $2.15 €$ & $21.50 €$ & häberle Shop & 9.207801 \\
\hline & \multicolumn{3}{|l|}{ Total } & \multicolumn{3}{|l|}{$1,095.72 €$} \\
\hline
\end{tabular}


The temporally discontinuous nature of rainfall poses a fundamental challenge to automatic rainwater sampling. In general, in order to prevent the pump from running dry and to avoid insufficient sample volumes during sample collection, rainwater needs to be pre-collected in a suitable container. In our case studies in karst caves we applied a specifically designed precollection container ("pre-collector") with an internal volume of exactly $12 \mathrm{~mL}$. During dripwater pre-collection a 3Dprinted floating body (volume considered) inside the pre-collector would rise until it seals the pre-collector once it is completely filled with dripwater. Any dripwater in excess of $12 \mathrm{~mL}$ spills over through a small hole at the top of the precollector (S6).

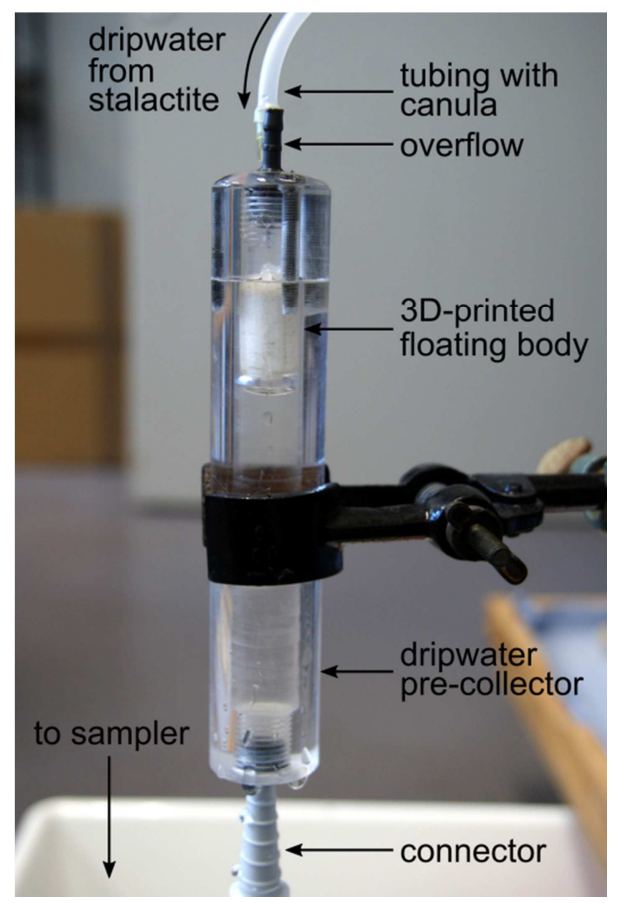

S7: Pre-collector used during the case studies.

\section{References}

Clark, I. D.; Fritz, P.: Environmental isotopes in hydrogeology. [2. print., corr.]. Boca Raton: Lewis Publ. 1999.

Hoefs, J.: Stable isotope geochemistry. 7. ed. Cham: Springer (Earth Sciences). 2015.

Smith, C. C., Löf, G., Jones, R.: Measurement and analysis of evaporation from an inactive outdoor swimming pool. In: Pergamon 1994, pp. 3-7. 1994. 